\title{
Factors on nodal up-staging in clinical NO adenocarcinoma patients who had minimally invasive anatomic lung resections
}

\author{
Tugba Cosgun ${ }^{1}$, Erkan Kaba', Kemal Ayalp², Alper Toker ${ }^{2}$ \\ 'Department of Thoracic Surgery, Demiroglu Bilim University, Istanbul 34381, Turkey. \\ ${ }^{2}$ Department of Thoracic Surgery, Istanbul Florence Nightingale Hospital, Istanbul 34381, Turkey.
}

Correspondence to: Dr. Tugba Cosgun, Sisli Florence Nightingale Hospital, Merkez Mah, Abide-i Hurriyet Cd No:164, Sisli/ Istanbul 34387, Turkey. E-mail: tugba_cosgun@hotmail.com

How to cite this article: Cosgun T, Kaba E, Ayalp K, Toker A. Factors on nodal up-staging in clinical NO adenocarcinoma patients who had minimally invasive anatomic lung resections. Mini-invasive Surg2019;3:32. http://dx.doi.org/10.20517/2574-1225.2019.024

Received: 17 Aug 2019 First Decision: 15 Oct 2019 Revised: 27 Oct 2019 Accepted: 5 Nov 2019 Published: 15 Nov 2019

Science Editor: Noriyoshi Sawabata Copy Editor: Jing-Wen Zhang Production Editor: Jing Yu

\begin{abstract}
Aim: The incidence of adenocarcinoma among lung cancer patients has increased in recent years. We identified the factors affecting lymph node status in patients with primary lung adenocarcinoma who underwent minimallyinvasive anatomic resection.
\end{abstract}

Methods: We retrospectively analyzed the medical records of primary lung adenocarcinoma patients who underwent minimally-invasive anatomic lung resections and mediastinal lymph node dissection between January 2012 and December 2017. We evaluated lymph node positivity and nodal status in each T and histologic subgroup, tumoral prognostic characteristics, minimally-invasive surgical methods and resection type.

Results: Of 473 patients who underwent anatomic resection for lung cancer between January 2012 and December 2017, 274 underwent minimally-invasive anatomic lung resections for primary lung cancer, 158 adenocarcinoma patients were analyzed in this study. Nodal status and number of positive lymph nodes were similar in the stages T1, T2, T3. Lymphovascular invasion ( $n: 78$ ) and micropapillary predominance tended to be significant predisposing factors for lymph node metastasis. Mean dissected lymph node number was significantly higher in patients who underwent Robot-assisted thoracoscopic surgery compared to Video-assisted thoracoscopic surgery $(P<0.05)$, and in those who underwent lobectomy compared to segmentectomy $(P<0.05)$.

\footnotetext{
(@) ( $)$

(C) The Author(s) 2019. Open Access This article is licensed under a Creative Commons Attribution 4.0 International License (https://creativecommons.org/licenses/by/4.0/), which permits unrestricted use, sharing, adaptation, distribution and reproduction in any medium or format, for any purpose, even commercially, as long as you give appropriate credit to the original author(s) and the source, provide a link to the Creative Commons license, and indicate if changes were made.
}

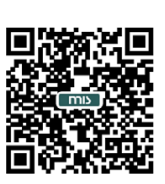


Conclusion: We were unable to demonstrate a relationship between $\mathrm{T}$ stage and $\mathrm{N}$ status. Factors contributing to unexpected $\mathrm{N}$ positivity were tumor characteristics that could not be identified in the preoperative period. We recommend performing systematic mediastinal lymph node dissection regardless of the size and histopathologic type of adenocarcinoma. In our study, robotic surgery and lobectomy operation showed superiority in dissecting more lymph nodes.

Keywords: Adenocarcinoma, robot-assisted thoracoscopic surgery, lymphovascular invasion, micropapillary predominance

\section{INTRODUCTION}

Lung cancer is one of the leading causes of death worldwide. Anatomic lung resections with systematic lymph node dissection have become the recommended treatment for early stage nonsmall lung cancer (NSCLC) $)^{[1]}$. Many investigators and clinicians recommend systematic nodal dissection to all lung cancer patients except those with clinical stage I disease ${ }^{[2]}$.

The Guidelines of the European Society for Medical Oncology (2014) recommend that preoperative invasive mediastinal staging (fine-needle aspiration with endobronchial ultrasonography/endoscopic ultrasonography guidance, or mediastinoscopy) should be used only if positive hilar nodes (stage N1 or $\mathrm{N} 2$ ) are suspected or tumor is located centrally on chest computed tomography (CT) or positron emission tomography $(\mathrm{PET}) / \mathrm{CT} \operatorname{scan}^{[3]}$. However, clinically diagnosed cNo disease preoperatively may sometimes be upstaged to N1 (pN1) or N2 (pN2) postoperatively ${ }^{[4]}$. Debate continues over whether systematic lymph node dissection is necessary for all patients with T1 or T2 tumors without signs of metastatic disease on preoperative clinical staging studies, such as CT-PET/CT, endobronchial ultrasound (EBUS), and endoscopic ultrasonography (EUS) ${ }^{[5,6]}$. Watanabe et al ${ }^{[7]}$ advocated that mediastinal nodal dissection would be unnecessary in patients with peripheral small lung cancers $(\leq 1 \mathrm{~cm}$ for adenocarcinomas and $2 \mathrm{~cm}$ for tumors other than adenocarcinoma). We speculated that adenocarcinoma subtypes are heterogeneous groups of lung cancers, and may even consist of mixed subtypes with different metastatic characteristics ${ }^{[8]}$. We also speculated that upstaging may be related to surgical technique and may change in the hands of the same surgeons with different surgical techniques.

Many studies have shown that the incidence of nodal upstaging postoperatively is a quality measure of surgery ${ }^{[4]}$. However, we speculated that upstaging may also be related to other characteristics of tumor in adenocarcinoma.

In this study, we tried to identify the relationships between tumor size, sub-histology, prognostic factors, and postoperative nodal upstaging in patients with clinical stage No adenocarcinoma who underwent minimally-invasive surgery.

\section{METHODS}

Of 274 patients who underwent minimally-invasive anatomic lung resections for primary lung cancer between January 2012 and December 2017, 158 (102 male, 56 female; mean age, $62.3 \pm 8.4$ years; range, 42-92 years) had clinical stage No primary lung adenocarcinoma and underwent minimally-invasive anatomic lung resections and systematic mediastinal lymph node dissections robotic assisted surgery (RATS; $n=83$ ) and video thoracoscopic surgery $(n=75)$. We retrospectively analyzed their prospectively collected medical records. A total of 17 patients were excluded because of insufficient data. Patients with positive mediastinal lymph nodes on mediastinoscopy and EBUS were underwent neoadjuvant treatment and excluded from the study. Patients who were diagnosed to have positive mediastinal lymph nodes 
preoperatively were excluded. Forty-one patients who underwent mediastinoscopy or EBUS preoperatively and patients who were reported as negative were included in this study. A total number of 158 patients without PET/CT, Mediastinoscopy, EBUS- evident stage N1 or N2 disease and those who underwent anatomic lung resections with systemic mediastinal and hilar complete lymph node dissections were analyzed. Mediastinal lymph node dissections yielded 1242 (mean, $18.8 \pm 10.6)$ lymph nodes with complete dissection of stations 5, 6, 7, 8, 9, 10, 11, 12 on the left, and 1919 lymph nodes (mean $20.9 \pm 11.8$ ) with complete dissection of stations $2 \mathrm{R}, 4 \mathrm{R}, 7,8,9,10,11$ on the right hemithorax.

\section{Surgical technique}

Patients were intubated and single-lung ventilation was placed via fiberoptic bronchoscopy. A lateral decubitus position was used. A two-port technique was used for videoassited thoracoscopic surgery (VATS) lobectomy. For RATS, three ports were opened, keeping $10 \mathrm{~cm}$ between each port and 10-15 $\mathrm{cm}$ from the target. We preferred to use the VATS-based approach for our RATS technique. When a fourth arm was used, it was placed lateral to the posterior arm. The technique we described here was used for Da Vinci SI systems (Da Vinci System Intuitive Surgical, Sunnyvale, CA, USA) ${ }^{[\rho]}$. The Da Vinci robotic system was used in 83 patients, while 75 underwent biportal VATS. Postoperative nodal status and number of positive lymph nodes were evaluated in each group and compared with each other.

Segmentectomy was preferred for tumors smaller than $2 \mathrm{~cm}$ and negative lymph nodes or for larger tumors in patients with poor pulmonary function who could not tolerate lobectomy, especially those who do not have visceral pleural invasion in our center. But if the tumor invaded visceral pleura or especially hilar lypmh node positivity could change the surgeon's decision. Also if the remaining tissue after segmentectomy seems not have a good ventilation or blood supply, we decided to perform completion lobectomy during the operation.

\section{Lymph node dissection}

Mediastinal and N1-level lymph node dissections were performed in similar manners on all patients regardless of whether they underwent RATS or VATS. Typically dissected mediastinal lymph node stations were $2 \mathrm{R}, 4 \mathrm{R}$, and 7-9 for patients with right-sided tumors, and 5-9 en bloc with perinodal fatty tissue for those with left-sided tumors. Segmentectomy became an operation option for peripheral, clinical T1NoMo, and $2 \mathrm{~cm}$ or smaller in size tumors. N1-level lymph nodes, and stations 10 and 11 for lobectomies, and additionally, station 12 were dissected completely and separately during segmentectomies. For segmentectomy, stations 11 and 12 nodes were dissected completely and evaluated by frozen section analysis $^{[10]}$. If either of them was positive, we preferred to perform lobectomy instead of segmentectomy.

Lymph node status and number of positive lymph nodes were evaluated for each patient. Rate of positive lymph nodes was calculated as the ratio of positive lymph nodes to the number of dissected lymph nodes.

\section{Histopathology}

Tumors were classified histologically as well (grade 1), medium (grade 2), and poorly (grade 3 ) differentiated. Histopathologic types of tumors also were analyzed. In 134 patients, the tumor could be categorized according to predominance of acinar, solid, lepidic, and micropapillary patterns.

Lymphovascular and visceral pleural invasion of tumors was analyzed according to positive lymph nodes and possible effects on $\mathrm{N}$ upstaging.

\section{Statistical analysis}

Lymph node positivity and nodal status were evaluated in each subgroup, comparing stages $\mathrm{T} 1$ vs. T2, T1 vs. T3, and T2 vs. T3. T stage subgroups, such as T1a, Tb, T1c, T2a, T2b, and T3, also were compared using 
Table 1. Characteristics of patients, tumors and operations

\begin{tabular}{ll}
\hline Patients' characteristics & $\boldsymbol{n}=\mathbf{1 5 8}(\%)$ \\
\hline Gender & $102(64.5 \%)$ \\
Male & $56(35.5 \%)$ \\
Female & \\
Side & $66(41.7 \%)$ \\
Left & $92(58.3 \%)$ \\
Right & \\
Type of operation & $32(20.25 \%)$ \\
Segmentectomy & 3 \\
Segment 6 & 1 \\
Segment 3 & 1 \\
Segment 1 & 5 \\
Segment 1-2 (left) & 2 \\
Common basal segmentectomy (7.8.9.10.) & 1 \\
Lingulectomy & 11 \\
Lingula sparing LUL & 8 \\
Segment 2 (right) & 126 (79.7\%) \\
Lobectomy & 27 \\
LUL & 21 \\
LLL & 53 \\
RUL & 8 \\
RML & 16 \\
RLL & 1 \\
Bilobectomy & \\
\hline & LUL: left upper lobectomy; LLL: left lower lobectomy; RUL: right upper lobectomy; RML: right \\
middle lobectomy; RLL: right lower lobectomy &
\end{tabular}

the Mann-Whitney $U$ and analysis of variance (ANOVA) tests. Regression of histopathologic subtypes and lymph node positivity, and nodal status were analyzed with ANOVA. Resection types (lobectomy, segmentectomy) and operative technique (VATS, RATS) also were analyzed with the Mann-Whitney $U$ test.

\section{RESULTS}

There were 158 patients in the study group with a mean age of $62.36 \pm 8.4$ (range 42-92). There were 102 male and 56 female patients. Characteristics of the patients, tumors and operations are noted in Table 1.

\section{Operative techniques}

Of the patients, 83 underwent RATS and 75 underwent VATS. Mean number of dissected lymph nodes was $23.3 \pm 11.3$ (range, 1-57) in the RATS group and $16.3 \pm 10.2$ (range, 2-49) in the VATS group. Robotic surgery showed higher numbers of lymph node dissections $(P<0.05)$. Mean number of positive lymph nodes was $1.2 \pm 2.6$ (range, 0 -11) in RATS group and $0.3 \pm 0.8$ (range, $0-4)$ in the VATS group $(P=0.06$ ). In final pathology 14 patients were staged as $\mathrm{N} 2,7$ patients were $\mathrm{N} 1$, and 62 were No in RATS group; 7 patients were N2, 4 patients were N1 and 64 were No in the VATS group $(P=0.13$; Table 2).

The mean number of dissected lymph nodes was $22.5 \pm 11.05$ in patients who underwent lobectomy, which was significantly higher than that in patients who underwent segmentectomy $(14.5 \pm 10.5 ; P<0.05)$. However, the numbers of positive lymph nodes were comparable between the groups ( $0.75 \pm 1.9$ vs. $0.6 \pm 1.9$, respectively; $P=0.36$ ).

\section{Tumor size}

According to the 8th Tumor, Nodes, and Metastases (TNM) classification, 108 patients had pathologic stage T1 (11 T1a, 69 T1b, 28 T1c), 34 stage T2 (23 T2a, 11 T2b), and 16 stage T3 tumors. A total of 26 patients 
Table 2. Type of minimal invasive surgery and effects of surgical technique on lymph nodes

\begin{tabular}{llll}
\hline & RATS 83 & VATS 75 & P value \\
\hline N of dissected LN/patients & $23.3 \pm 11.3$ & $16.3 \pm 10.2$ & $P<0.05$ \\
N of positive LN/patients & $1.2 \pm 2.6$ & $0.3 \pm 0.8$ & $P=0.06$ \\
N status & & & \\
N2 & $14(17 \%)$ & $7(9.3 \%)$ & $P=0.13$ \\
N1 & $7(8.4 \%)$ & $4(5.3 \%)$ & \\
NO & $62(74.7 \%)$ & $64(85.3 \%)$ & \\
\hline
\end{tabular}

N: number; LN: lymph node; RATS: robot assisted thoracoscopic surgery; VATS: videoassited thoracoscopic surgery; N status: nodal status ( $P$ value was derived from ANOVA test)

Table 3. Effects of $\mathrm{T}$ factor on nodal status and positivity of lymph nodes

\begin{tabular}{|c|c|c|c|c|c|c|c|}
\hline & \multicolumn{3}{|c|}{ T1 } & \multicolumn{2}{|c|}{ T2 } & \multirow{2}{*}{$\begin{array}{l}\text { T3 } \\
\text { T3 }\end{array}$} & \multirow{2}{*}{$P$ value } \\
\hline & T1a & T1b & T1c & T2a & T2b & & \\
\hline Number of patients & 11 & 69 & 28 & 23 & 11 & 16 & \\
\hline NO & $8 \%-72.7 \%$ & $56 \%-81.2 \%$ & $22 \%-78.6 \%$ & $18 \%-78.26 \%$ & $10 \%-90.1 \%$ & $12 \%-75 \%$ & \\
\hline $\mathrm{N} 1$ & $1 \%-9 \%$ & $5 \%-7.3 \%$ & $1 \%-3.6 \%$ & $3 \%-13 \%$ & $1 \%-9 \%$ & 0 & $P>0.05$ \\
\hline N2 & $2 \%-18 \%$ & $8 \%-13.3 \%$ & $5 \%-17.8 \%$ & $2 \%-8.6 \%$ & 0 & $4 \%-25 \%$ & \\
\hline Number of positive lymph nodes & 6 & 46 & 23 & 20 & 1 & 21 & $P>0.05$ \\
\hline Rate of positive lymph nodes & 0.03 & 0.045 & 0.034 & 0.03 & 0.033 & 0.042 & $P>0.05$ \\
\hline
\end{tabular}

$P$ value was derived from ANOVA test

had multifocal tumors. Mean tumor size was $2.8 \pm 1.9 \mathrm{~cm}$ (range, $0.6-11 ; 2.9 \pm 2$ in the RATS and $2.6 \pm 1.8$ in the VATS groups; $P=0.4$ ).

\section{Lymph nodes}

The most commonly dissected lymph nodes were those at stations 5-7, 10, and 11 for left-sided tumors, and at stations $2 \mathrm{R}, 4 \mathrm{R}$, and 7-11 for right-sided tumors. Mean number of dissected lymph nodes was $20.0 \pm 11.3$ (range, 1-57).

Among all patients, the nodal status was N2 in 21 (13.3\%), N1 in 11 (7\%), and No in 126 (79.\%). Total upstaging rate in all groups was $20.3 \%$.

Nodal status and number of positive lymph nodes were evaluated with the Mann-Whitney $U$ test for stages T1 vs. T2 $(P=0.79, P=0.77)$, T1 vs. T3 $(P=0.32, P=0.36)$, and T2 vs. T3 $(P=0.29, P=0.39)$ disease. All differences were statistically insignificant. Nodal status and number of positive lymph nodes also were not statistically significant when comparing the three stage (T1, T2, T3) groups via ANOVA [Table 3].

Subgroups of T status (T1a, T1b, T1c, T2a, T2b, and T3) were compared and they had similar numbers of positive lymph nodes, positive lymph node ratios, and similar nodal status [Table 3].

\section{Histopathology}

Lymphovascular and visceral pleural invasions are the most commonly investigated histopathologic parameters. A total of 77 patients had lymphovascular and 34 had visceral pleural invasion. When analyzed, the effect of visceral pleural invasion on lymph node positivity was not significant $(P=0.29)$. However, lymphovascular invasion tended to be significantly related to lymph node positivity $(P=0.08)$.

Histologic tumor grade was classified according to differentiation: 16 patients had grade 1, 86 grade 2, and 41 grade 3 disease. There was no correlation between differentiation grade and lymph node positivity $(P=$ $0.86)$ and $\mathrm{N}$ status $(P=0.79)$. 
Histopathologic tumor types and their effect on lymph node positivity also were analyzed retrospectively. Of the patients, 134 could be categorized according to predominance of acinar, solid, lepidic, and micropapillary patterns. Micropapillary histologic subtype was associated with lymph node positivity. Other subtypes did not show significance $(P=0.65, P=0.22, P=0.78, P=0.005$, respectively).

\section{DISCUSSION}

Lymphatic dissemination is the major route of systematic metastasis, and it is the major determinant of long-term patient outcome. When a patient with clinical stage No disease has been demonstrated to have $\mathrm{N} 1$ or N2 positivity, not only prognosis, but also treatment modalities change. Larger consolidation size, central tumor location, and clinical N1-N2 stage have been defined as predictors of mediastinal lymph node metastasis ${ }^{[11]}$. A recent study demonstrated a significant increase in nodal upstaging to be related with the duration between radionuclear evaluation and surgery ${ }^{[12]}$.

A current study shows that $<2 \mathrm{~cm}$ tumors may show occult nodal metastasis, so that dissection of lymph nodes during sublobar resection increases survival for patients underwent sublobar resection ${ }^{[13]}$. The same study claimed that nonanatomic resections without hilar lymphadenectomy may miss this upstaging. Preoperative radiographic tumor size, tumors not in the upper lobe, high carcinoembryonic antigen levels, and micropapillary predominant adenocarcinomas were identified as predictors for unexpected $\mathrm{N} 1$ or $\mathrm{N} 2$ node positivity in adenocarcinoma patients ${ }^{[14]}$. When two major types (squamous cell carcinoma and adenocarcinoma) were compared, lymph node metastasis occurred more frequently in adenocarcinomas than in squamous cell carcinomas and it was reported to be uncommon for mediastinal lymph node metastasis in tumors with a diameter $<3 \mathrm{~cm}$. Poor differentiation grade may have an important role in lymph node metastasis ${ }^{[15]}$. Despite these findings, several investigators claim that mediastinal lymph node dissection may be unnecessary for adenocarcinomas $\geq 1 \mathrm{~cm}^{[7]}$. In our study, patients with clinicaly No disease, had postoperatively $\mathrm{N}$ stages $(\mathrm{N} 1, \mathrm{~N} 2)$ as high as $7.4 \%$ and $12.3 \%$ of cases, respectively, among all those with stages T1a and Tab cancer. Stage T1c group demonstrated similar upstaging (3.5\% patient was $\mathrm{N} 1$ disease and $17.8 \%$ patient was N2 disease postoperatively). Our results contradicted the literature findings and recommendations. We recommended mediastinal and hilar systematic lymph node dissection to be performed for all stages, including stage $1 \mathrm{~A}$.

In another trial on adenocarcinomas $\leq 30 \mathrm{~mm}$, solid pattern, maximum standardized uptake value, and lymphovascular invasion were independent predictors for lymph node metastasis ${ }^{[16]}$.

Adenocarcinoma is a heterogeneous type of lung carcinoma and mostly consists of mixed subtypes. According to one study on solitary peripheral subsolid nodules; speculation, lesion size, vascular convergence and solid proportion are predictive parameters of invasive adenocarcinoma ${ }^{[17]}$. In another study that used nomogram for predicting risk of invasive pulmonary adenocarcinoma for pure groundglass opacity nodules; lesion size, speculation, lobulation, air bronchogram, vascular convergence, pleural tag were risk factors for being invasive pulmonary adenocarcinoma ${ }^{[18]}$.

Adenocarcinoma in situ and minimally-invasive adenocarcinoma, in which a lepidic pattern is a major component, may show very good prognosis without mediastinal and hilar lymph node metastasis ${ }^{[19]}$. Lepidic predominant, minimally-invasive adenocarcinoma, and adenocarcinoma in situ also are specified as safe tumors for lymph node invasion ${ }^{[20,21]}$. Since a lepidic pattern is known to be noninvasive and often appears as a ground glass opacity (GGO) on radiologic evaluation, and a patient with a GGO nodule on tomography is likely to be diagnosed with adenocarcinoma in situ or minimally-invasive adenocarcinoma pathologically, systematic lymph node dissection might not be considered ${ }^{[19]}$. Lepidic predominance has been shown to be the safest subtype in regard to mediastinal and hilar lymph nodes in our study $(P=0.78)$, similar to findings in the literature. 
Moon et al ${ }^{[19]}$ claimed that mediastinal lymph node dissection may not be necessary for clinical stage No NSCLC presenting with $a \leq 3 \mathrm{~cm}$ GGO-predominant nodule. Segmentectomy can be a preferred technique if there is almost no lymph node metastasis at stations 12 and 11. In patients with adenocarcinoma and a micropapillary or solid component, lobectomy should be considered because of possible interlobar and intralobar lymph node metastasis ${ }^{[22]}$, which may not be identified before and during surgery. A histologic component may be an important factor in patients with nodal upstaging of clinically No tumors ${ }^{[4]}$. It has been shown that micropapillary and solid tumor patterns significantly increase the risk of nodal upstaging ${ }^{[23,24]}$. Our study demonstrated that the micropapillary pattern is related to a higher rate of lymph node positivity. However, we were not able to identify solid predominance as a risk factor for lymph node positivity.

Spread through air spaces (STAS) was defined as spread of lung cancer tumor cells into air spaces in the lung parenchyma adjacent to the main tumor. Three morphologic patterns of STAS were identified: (1) micropapillary structures, consisting of papillary structures without central fibrovascular cores that occasionally form ring-like structures within air spaces; (2) solid nests or tumor islands, consisting of solid collections of tumor cells filling air spaces; and (3) single cells consisting of scattered discohesive single cells $^{[25]}$. Lymphovascular invasion and high-grade morphologic pattern were identified more frequently in STAS-positive than STAS-negative tumors. Besides this, the risk of locoregional or distant recurrence was significantly higher in patients with STAS-positive than STAS-negative tumors who underwent limited resection. However, this association was not noted in the lobectomy group ${ }^{[26]}$.

Another remarkable finding of this study was the number of dissected lymph nodes. The number of positive and total number of dissected lymph nodes were higher in the RATS compared to the VATS groups $(P=0.06, P<0.05$, respectively). These results were compatible with those of previous studies from our department ${ }^{[27]}$. We believed that RATS increases the capability of mediastinal dissection. On the other hand, our group has been performing VATS lobectomy consistently for more than a decade; we do not see this finding in VATS.

Limitation of our study are as follows: this study is performed in a single center with relatively small size population. We could not analyze the patients with preoperatively diagnosed N2 disease. Relatively a small number of patients underwent mediastinoscopy and/or or EBUS preoperatively in our series.

The number of positive lymph nodes and lymph node status were comparable between the $\mathrm{T}$ groups and subgroups in adenocarcinomas. These results may be related with heterogenous types and subgroups of adenocarcinoma. We demonstrated that lymphovascular invasion and micropapillary predominance could be considered candidates for nodal upstaging. Since these features of tumors cannot be identified preoperatively in most patients, we recommended performing systematic hilar and mediastinal lymph node dissection for an adenocarcinoma of any size considering minimally-invasive surgery; we preferred the robotic-assisted approach.

\section{DECLARATIONS}

\section{Authors' contributions}

Conceived and designed the study, wrote and reviewed the manuscript: Cosgun $\mathrm{T}$

Collected and tabulated data, participated in manuscript writing: Kaba E, Ayalp K

Participated in manuscript writing and review: Toker A

\section{Availability of data and materials}

Not applicable. 


\section{Financial support and sponsorship}

None.

\section{Conflicts of interest}

All authors declared that there are no conflicts of interest.

\section{Ethical approval and consent to participate}

Not applicable.

\section{Consent for publication}

Not applicable.

\section{Copyright}

(C) The Author(s) 2019.

\section{REFERENCES}

1. Yu X, Li Y, Shi C, Han B. Risk factors of lymph node metastasis in patients with non-small cell lung cancer $\leq 2 \mathrm{~cm}$ in size: A monocentric population-based analysis. Thorac Cancer 2018;9:3-9.

2. Takizawa H, Kondo K, Matsuoka H, Uyama K, Toba H, et al. Effect of mediastinal lymph nodes sampling in patients with clinical stage I non-small cell lung cancer. J Med Invest 2008;55:37-43.

3. Postmus PE, Kerr KM, Oudkerk M, Senan S, Waller DA, et al. Early and locally advanced non-small-cell lung cancer (NSCLC): ESMO clinical practice guidelines for diagnosis, treatment and follow-up. Ann Oncol 2013;24:89-98.

4. Moon Y, Kim KS, Lee KY, Sung SW, Kim YK, et al. Clinicopathologic factors associated with occult lymph node metastasis in patients with clinically diagnosed N0 lung adenocarcinoma. Ann Thorac Surg 2016;101:1928-35.

5. Ettinger DS, Akerley W, Borghaei H, Akerley W, Bauman J, et al. Non-small cell lung cancer. J Natl Compr Canc Netw 2012;10:1236-71.

6. Gulack BC, Yang CF, Speicher PJ, Meza JM, Gu L, et al. The impact of tumor size on the association of the extent of lymph node resection and survival in clinical stage I non-small cell lung cancer. Lung Cancer 2015;90:554-60.

7. Watanabe S, Oda M, Go T, Ohta Y, Watanabe Y, et al. Should mediastinal nodal dissection be routinely undertaken in patients with peripheral small-sized ( $2 \mathrm{~cm}$ or less) lung cancer? Retrospective analysis of 225 patients. Eur J Cardiothorac Surg 2001;20:1007-11.

8. Travis WD, Brambilla E, Noguchi M, Nicholson AG, Geisinger K, et al. International association for the study of lung cancer/ American thoracic society/European respiratory society international multidisciplinary classification of lung adenocarcinoma. J Thorac Oncol 2011;6:244-85.

9. Toker A, Kaba E, Ayalp K, Ozyurtkan MO. Robotic lung resections: video-assisted thoracic surgery based approach. J Vis Surg 2017;3:15.

10. Toker A, Ozyurtkan MO, Demirhan O, Ayalp K, Kaba E, et al. Lymph node dissection in surgery for lung cancer: Comparison of open vs. video-assisted vs. robotic-assisted approaches. Ann Thorac Cardiovasc Surg 2016;22:284-90.

11. Zang RC, Qiu B, Gao SG, He J. A model predicting lymph node status for patients with clinical stage TlaN0-2M0 nonsmall cell lung cancer. Chin Med J (Engl) 2017;130:398-403.

12. Gard G, Voskoboynik M. Delay to Surgery May Upstage Patients With Non-Small Cell Lung Cancer. Chest 2019;156:633-4. doi: 10.1016/j.chest.2019.04.113.

13. Stiles BM, Mao J, Harrison S, Lee B, Port JL et al. Extent of lymphadenectomy is associated with oncological efficacy of sublobar resection for lung cancer $\leq 2 \mathrm{~cm}$. J Thorac Cardiovasc Surg 2019;157:2454-65.

14. Bao F, Yuan P, Yuan X, Lv X, Wang Z, et al. Predictive risk factors for lymph node metastasis in patients with small size non-small cell lung cancer. J Thorac Dis 2014;6:1697-703.

15. Pan T, Zheng Z, Li J, Tang Y, Pan Y, et al. Relationship between tumor size and lymph node metastasis in squamous cell carcinoma and adenocarcinoma of the lung. Zhongguo Fei Ai Za Zhi 2006;9:267-9.

16. Seok Y, Yang HC, Kim TJ, Lee KW, Kim K, et al. Frequency of lymph node metastasis according to the size of tumors in resected pulmonary adenocarcinoma with a size of $30 \mathrm{~mm}$ or smaller. J Thorac Oncol 2014;9:818-24.

17. Jin C, Cao J, Cai Y, Wang L, Liu K, et al. A nomogram for predicting the risk of invasive pulmonary adenocarcinoma for patients with solitary peripheral subsolid nodules. J Thorac Cardiovasc Surg 2017;153:462-9.

18. Wang L, Shen W, Xi Y, Liu S, Zheng D, et al. Nomogram for predicting the risk of invasive pulmonary adenocarcinoma for pure ground-glass nodules. Ann Thorac Surg 2018;105:1058-64.

19. Moon Y, Sung SW, Namkoong M, Park JK. The effectiveness of mediastinal lymph node evaluation in a patient with ground glass opacity tumor. J Thorac Dis 2016;8:2617-25.

20. Yu Y, Jian H, Shen L, Zhu L, Lu S. Lymph node involvement influenced by lung adenocarcinoma subtypes in tumor size $\leq 3 \mathrm{~cm}$ disease: A study of 2268 cases. Eur J Surg Oncol 2016;42:1714-9. 
21. Jiang L, Yin W, Peng G, Wang W, Zhang J, et al. Prognosis and status of lymph node involvement in patients with adenocarcinoma in situ and minimally invasive adenocarcinoma-a systematic literature review and pooled-data analysis. J Thorac Dis 2015;7:2003-9.

22. Wang L, Jiang W, Zhan C, Shi Y, Zhang Y, et al. Lymph node metastasis in clinical stage IA peripheral lung cancer. Lung Cancer 2015;90:41-6.

23. Yuan Y, Ma G, Zhang Y, Chen H. Presence of micropapillary and solid patterns are associated with nodal upstaging and unfavorable prognosis among patient with cT1N0M0 lung adenocarcinoma: a large-scale analysis. J Cancer Res Clin Oncol 2018;144:743-9.

24. Yu Y, Jian H, Shen L, Zhu L, Lu S. Lymph node involvement influenced by lung adenocarcinoma subtypes in tumor size $\leq 3 \mathrm{~cm}$ disease: A study of 2268 cases. Eur J Surg Oncol 2016;42:1714-9.

25. Onozato ML, Kovach AE, Yeap BY, Morales-Oyarvide V, Klepeis VE, et al. Tumor islands in resected early-stage lung adenocarcinomas are associated with unique clinicopathologic and molecular characteristics and worse prognosis. Am J Surg Pathol 2013;37:287-94.

26. Kadota K, Nitadori J, Sima CS, Ujiie H, Rizk NP, et al. Tumor spread through air spaces is an important pattern of invasion and impacts the frequency and location of recurrences after limited resection for small stage I lung adenocarcinomas. J Thorac Oncol 2015;10:806-14.

27. Toker A, Ozyurtkan MO, Demirhan O, Ayalp K, Kaba E, et al. Lymph node dissection in surgery for lung cancer: Comparison of open vs. video-assisted vs. robotic-assisted approaches. Ann Thorac Cardiovasc Surg 2016;22:284-90. 